\title{
Cinémas
}

Revue d'études cinématographiques

Journal of Film Studies

\section{Incohérence et transgression dans Sucker Punch : une esthétique du secret. Lecture phénoménologique d'un (dé)voilement du sens}

\section{Incoherence and Transgression in Sucker Punch: An Aesthetic of the Secret. A Phenomenological Reading of an (un)Veiling of Meaning}

\section{Matthieu Dubois}

Volume 25, numéro 2-3, printemps 2015

URI : https://id.erudit.org/iderudit/1035776ar

DOI : https://doi.org/10.7202/1035776ar

Aller au sommaire du numéro

Éditeur(s)

Cinémas

ISSN

1181-6945 (imprimé)

1705-6500 (numérique)

Découvrir la revue

Citer cet article

Dubois, M. (2015). Incohérence et transgression dans Sucker Punch : une esthétique du secret. Lecture phénoménologique d'un (dé)voilement du sens. Cinémas, 25(2-3), 141-158. https://doi.org/10.7202/1035776ar
Résumé de l'article

Cet article prend le parti de lire Sucker Punch (Coup interdit, 2011), de Zack Snyder, à contre-courant des critiques qui n'y ont vu qu'un film miné par des incohérences et un scénario sans profondeur. À partir de l'analyse d'un délire diégétique, l'auteur met en lumière des indices qui l'amènent à élaborer une interprétation à rebours du récit, sur le modèle de l'analyse qu'effectue Pierre Bayard (1998) du Meurtre de Roger Ackroyd d'Agatha Christie. Avec l'aide de la phénoménologie de Michel Henry est mise au jour une réflexion sur le caractère nécessairement subjectif, trompeur, de la narration filmique, qui invite à concevoir un twist structurant le récit : soit à considérer l'existence de l'héroïne (Babydoll) comme illusoire au profit d'un personnage secondaire (Sweet Pea), selon un procédé de dédoublement de la personnalité visant à mettre à distance un vécu traumatique. Cela fait de Sucker Punch une oeuvre qui interroge en profondeur les potentialités de l'imaginaire et propose, en creux, une réflexion sur les enjeux du septième art, en cette ère de développement sans précédent des effets numériques. 


\title{
Incohérence et transgression dans Sucker Punch: une esthétique du secret. Lecture phénoménologique d'un (dé)voilement du sens
}

\section{Matthieu Dubois}

\begin{abstract}
RÉSUMÉ
Cet article prend le parti de lire Sucker Punch (Coup interdit, 2011), de Zack Snyder, à contre-courant des critiques qui n'y ont vu qu'un film miné par des incohérences et un scénario sans profondeur. À partir de l'analyse d'un délire diégétique, l'auteur met en lumière des indices qui l'amènent à élaborer une interprétation à rebours du récit, sur le modèle de l'analyse qu'effectue Pierre Bayard (1998) du Meurtre de Roger Ackroyd d'Agatha Christie. Avec l'aide de la phénoménologie de Michel Henry est mise au jour une réflexion sur le caractère nécessairement subjectif, trompeur, de la narration filmique, qui invite à concevoir un twist structurant le récit: soit à considérer l'existence de l'héroïne (Babydoll) comme illusoire au profit d'un personnage secondaire (Sweet Pea), selon un procédé de dédoublement de la personnalité visant à mettre à distance un vécu traumatique. Cela fait de Sucker Punch une œuvre qui interroge en profondeur les potentialités de l'imaginaire et propose, en creux, une réflexion sur les enjeux du septième art, en cette ère de développement sans précédent des effets numériques.
\end{abstract}

Selon l'interprétation de la littérature comme rite développée par Myriam Watthee-Delmotte, la réception d'une œuvre s'accomplit entre autres grâce à la mise en place d'une stratégie qui vise à fonder, dans l'imaginaire, un échange entre l'écrivain et le lecteur. Aussi un texte déploie-t-il des codes destinés à être repérés et sur lesquels repose sa performativité :

[...] l'auteur propose une structure rituelle qui, pour une part, repose sur des antécédents éprouvés (les formes dénombrées par l'histoire littéraire) et, pour une autre part, fait jouer sa différence 
(c'est l'espace de liberté du créateur qui motivera pour une part le choix de son ouvre par le lecteur) (Watthee-Delmotte 2010, p. 69-70)

De ce point de vue, l'accueil de l'œuvre fait l'objet d'un pari qui, en tant que tel, n'est jamais assuré d'aboutir.

Cette approche rituelle de la création littéraire peut s'appliquer également au cinéma. La réception du film Sucker Punch (Coup interdit, Zack Snyder, 2011) rappelle les difficultés qu'a connues la pièce de Pierre Corneille Lillusion comique (1636), qui déploie une poétique de l'irrégularité fondée sur une structure narrative complexe ${ }^{1}$. Les critiques du film révèlent de façon similaire la déception d'un certain horizon d'attente. Le réalisateur transgresse volontiers les codes cinématographiques au profit de l'émergence d'une esthétique audacieuse, au point de craindre, avoue-t-il lui-même, "de paraître trop bizarre, trop dégénéré pour le propre bien du film et donc d'exclure ceux qui ne sont pas familiers avec le genre» (Snyder 2011). En se réappropriant la culture populaire des films d'action, de la fantasy et des jeux vidéo, le cinéaste américain fait droit à une jouissance des possibles visuels à l'encontre d'une approche plus "classique ", laquelle tend, par ailleurs, à cloisonner les genres ${ }^{2}$.

Alors qu'une communauté importante de cinéphiles lui reproche, entre autres, un scénario sans profondeur, Snyder (2011) invite à l'opposé à «voir le film à répétition pour distinguer ce qui tient du réel et de l'imaginaire». On peut dès lors postuler que la recherche d'une complexité à la fois esthétique et diégétique s'est traduite par l'opacification du sens. Bien que le film soit considéré comme mineur, il s'agit d'en faire une lecture au profit d'une clef interprétative dérobée, afin de lui restituer sa richesse ${ }^{3}$.

\section{Du délire psychologique au délire diégétique}

Sucker Punch met en scène une adolescente, Babydoll, aux prises avec un univers psychiatrique aliénant, dont elle cherche à s'évader en entraînant avec elle quatre autres jeunes filles: Sweet Pea, Rocket, Amber et Blondie. L'histoire se conçoit à la fois comme un récit initiatique et comme un récit d'évasion dans la tradition du «film de prison avec des détenus qui veulent s'extraire d'un milieu carcéral» (Snyder 2011). 
Babydoll se retrouve enfermée à l'institut Lennox contre son gré pour le meurtre de sa sœur, qu'elle a tuée accidentellement alors que toutes deux étaient menacées de violence par leur beau-père. Celui-ci masque les faits et passe un arrangement crapuleux avec l'infirmier-chef de l'asile pour faire lobotomiser Babydoll rapidement. Durant le temps qu'il lui reste avant l'opération, la jeune fille se construit un délire, qu'elle partage avec ses amies, dans lequel l'asile est transfiguré en cabaretbordel où les patientes incarnent des danseuses qui s'entraînent à séduire les clients de Blue Jones, l'alter ego de l'infirmier-chef reconverti en gérant et maquereau. C'est dans cet univers imaginaire que les héroïnes préparent leur évasion, laquelle nécessite cinq éléments: pour se les procurer, les jeunes filles pénètrent, de façon ponctuelle, dans un second niveau imaginaire, où elles vivent des aventures dantesques qui amplifient de façon hyperbolique le caractère héroïque du vol des objets.

Sucker Punch déploie un système d'emboîtements diégétiques dont la structure vient se substituer au réel. La linéarité du récit qui conduit Babydoll de son enfermement à la lobotomie s'enrichit d'une superposition de niveaux qui confère au film une pluridimensionnalité tant esthétique qu'événementielle. L'institut Lennox se présente comme un environnement implacable, une sorte de purgatoire où les patientes, soumises à des corvées dégradantes et aux violences sexuelles des infirmiers, attendent la lobotomie comme une délivrance qui les fera accéder au "paradis". Le passage dans le monde du cabaret opère la transfiguration de l'institut psychiatrique - espace clos caractérisé par son aspect décrépit, froid, sans ouverture sur l'extérieur - en un lieu chamarré, caractéristique d'un univers baroque: les costumes des patientes y sont transformés en tenues légères, la psychiatre Vera Gorski y incarne une prostituée-chorégraphe et le médecin qui pratique les lobotomies y devient le mystérieux High Roller désireux de "consommer" la chair de Babydoll. Cet univers est également régi par des règles de violence, figurées par l'autorité de Blue (qui n'hésite pas à abattre les danseuses qui lui désobéissent), ainsi que par la nécessité pour les jeunes filles de s'exposer aux regards lorsqu'elles dansent. On notera la filiation sémantique entre l'asile et le cabaret-bordel, qui tous deux représentent 
des "maisons closes", aux sens propre et figuré, et impliquent l'aliénation. C'est seulement au deuxième niveau imaginaire - qui s'apparente à un monde de fantasy multiforme, inspiré tant par les romans de Tolkien ${ }^{4}$ que par la science-fiction contemporaine et le fantastique - que les héroïnes peuvent réparer pleinement leurs blessures narcissiques, en incarnant des combattantes qui triomphent des figures archétypiques du mal dans des décors à ciel ouvert. Cet univers développe à outrance le "régime diurne" des imaginaires analysés par Gilbert Durand (1960), caractérisés par le rapport manichéen aux valeurs que symbolise leur arsenal médiéval et moderne.

Le film exprime l'intériorité délirante qui projette ses fantasmes sur le réel. Toutefois, il ne s'agit pas d'explorer la folie, mais de rendre compte de la puissance cathartique de la mise en scène de soi, laquelle est liée aux méthodes de la psychiatre qui tente de soigner ses patientes en les exhortant à refigurer leur vécu: "Tu contrôles ce monde. Chasse la douleur. Chasse la souffrance. Chasse la culpabilité." Le film donne à voir la mise en ouvre d'une thérapie qui vise à réinscrire le sujet dans un mieux-être possible à travers la fictionnalisation de soi. La jonction entre le réel (l'institut) et le fantasmé (le cabaret-bordel) se produit au moment de la lobotomisation de Babydoll: l'opération est détournée sur le mode du pastiche pour s'inscrire dans le cadre d'une répétition de spectacle qui en atténue la charge menaçante par sa mise à distance ludique. En dépit des violences exercées dans le cabaret-bordel, ce quotidien s'avère plus valorisant que celui de l'asile: avec la danse se joue la possibilité d'agir, plutôt que de subir, et d'accéder ainsi à une forme d'accomplissement de soi.

Le film, qui montre la puissance de refiguration de l'imaginaire, révèle une contamination de la narration par le délire des personnages, dont les mondes imaginaires développent une poétique de l'incohérence fondée sur la profusion des images agencées en récits trépidants et sur le déploiement d'une théâtralité paroxystique composée de ralentis et de sons intensifiés ${ }^{5}$. À quoi s'ajoute le détournement d'événements historiques par l'insertion d'éléments fantastiques (les soldats allemands de la Première Guerre mondiale sont des zombies animés par des 
boulons et de la vapeur) ou anachroniques (des armes à feu récentes, voire futuristes, figurent dans la reconstitution historique d'une bataille).

Au-delà de la relation entre esthétique cinématographique et projections fantasmatiques, on remarque la présence de paradoxes qui fragilisent l'œuvre. On peut repérer des effets de contradiction, de décalage: le surnom "Blondie» ne correspond pas au physique de brune du personnage, de même que celui de «Babydoll» ne dit rien de la force guerrière de la jeune fille. Par ailleurs, Zack Snyder montre de façon cynique la fascination qu'exerce l'univers sinistre de l'asile - ce qui donne lieu à des oxymores visuels, tels les stéréotypes de femmes à la fois soumises au désir des hommes et guerrières invincibles. Le film présente en outre plusieurs incohérences diégétiques: lors du passage au premier niveau imaginaire, comment comprendre le changement de perspective (Sweet Pea substituée à Babydoll) et le fait que la scène rejouée constitue une prolepse? Comment comprendre le brouillage des différents niveaux de réalité dans la scène finale, lorsque Sweet Pea croise deux figures qui appartiennent aux mondes de fantasy et deux policiers entrevus dans la première scène?

Ces éléments qui surgissent de façon imprévisible au sein d'une narration prévisible - fondée sur l'alternance mécaniquement rythmée des différents niveaux imaginaires — s'expliquent par une porosité entre délire intérieur et réel, de sorte que le film se révèle lui-même délire diégétique. Cette fragilité de la scénographie se trouve à l'origine des critiques dont Sucker Punch a fait l'objet, et qui font état de faiblesses tant dans l'écriture du scénario que dans le montage du film.

\section{Voilement/dévoilement du sens}

L'un des problèmes qui touchent la réception du film concerne l'indétermination de la réalité des événements. Les différents niveaux imaginaires constituent autant de filtres qui se superposent à l'épreuve de l'asile et dissimulent certains faits — en raison de leur caractère indicible — en les refigurant. Ainsi, la tactique que les héroïnes mettent au point pour s'évader repose sur la diversion que produit la danse, assimilable à 
une transe, de Babydoll; celle-ci captive toute l'attention des spectateurs au point de les hypnotiser; cependant ses gestes demeurent seulement esquissés, aussitôt traduits par une chorégraphie guerrière fantasmatique. Sucker Punch repose donc sur ce que Michel Collot, dans son étude sur la poésie moderne, appelle la "structure d'horizon", dès lors que ce qui s'y joue réellement est dérobé au spectateur: pour le réalisateur comme pour le poète, il ne s'agit pas de "défigurer pour le rendre méconnaissable un sens qu['il] pourrait se figurer autrement, mais de donner forme à l'infigurable»(Collot 1989, p. 246).

L'inscription de l'horizon au cœur de l'image implique un retrait de son pouvoir, selon un mouvement paradoxal de démonstration spectaculaire des effets numériques - capables de faire voir l'impossible - qui vient en retour faire écran. Les images ne visent pas, ici, à capter le réel, mais à "rend[re] la chose inépuisable" (Collot 1989, p. 179). Cette visée implique un processus de "voilement» de la réalité, qui prive le spectateur de la satisfaction de la pulsion scopique et produit un voilement du sens. En contrepoint, Sucker Punch invite le spectateur à dépasser le donné visuel en livrant des indices à double entente qui peuvent mettre sur la voie d'une quête de sens. Le Sage qui guide Babydoll et ses compagnes mentionne "une énigme» $(a$ mystery), laquelle constitue la finalité du récit: "C'est la raison. Le but. " L'œuvre thématise de la sorte un dire à décrypter dans ses éléments.

Le spectateur se trouve alors placé dans la posture du lecteur, entendu dans le sens originaire, évoqué par Daniel Ferrer (1998, p. 15-16), de celui qui déchiffre pour reconstituer le fil de ce qui s'est passé. La scène qui suit le moment où Babydoll est lobotomisée, après l'évasion de Sweet Pea, est indicative de cette situation. Ce passage marque le retour à la "réalité rugueuse» de l'asile en révélant, sur le mode de l'indice, une partie des dégâts causés par les cinq amies dans leur délire partagé; ces traces ancrent le délire, en ce qu'il peut motiver une véritable action. Ainsi, Sucker Punch produit, à l'instar de l'œuvre littéraire, ses propres mécanismes de mobilisation du sujet. Comme le rappelle Umberto Eco (1979, p. 84), «un texte est un artifice syntaxico-sémantico-pragmatique dont l'interprétation prévue 
fait partie de son propre projet génératif", de sorte que le lecteur est invité à prendre une part active à l'élaboration de l'œuvre. De façon comparable, le spectateur doit participer à l'actualisation des potentialités du film pour en jouir pleinement.

On peut remarquer de ce point de vue la présence de prolepses qui soulignent la cohérence interne du récit. Les armes de Babydoll - un katana et un pistolet - sont évoquées anticipativement dans la première scène lorsque le beau-père décachette le testament de sa femme. Le choix du sabre de samouraï fait en outre référence aux valeurs, fondées sur le don de soi, associées à la caste dont il est le symbole et s'avère ainsi programmatique du sacrifice final de la jeune fille. Le lapin en peluche de la sœur de Babydoll apparait par la suite en pendentif sur le pistolet de l'héroïne, ainsi que sur l'exosquelette d'Amber. À cela s'ajoutent des échos formels: l'architecture de l'asile psychiatrique transparaît dans les bâtiments des mondes imaginaires — qu'il s'agisse du cabaret-bordel ou de la cathédrale en ruine de la séquence parodique de la Première Guerre mondiale, ou encore du château de la séquence fantastico-médiévale. Enfin, parmi les multiples exemples que l'on peut relever, le surnom "Babydoll ${ }^{6}$ " prédestine celle qui le porte à la lobotomie qui la transformera en marionnette.

Se dessine alors une porosité entre les différentes sphères diégétiques, qui entretiennent des liens de contiguïté sémantique ou symbolique. Ceux-ci ne sont pas systématiquement élucidés; le spectateur n'a accès que de façon fragmentaire aux événements qui ont eu des conséquences tangibles, tels l'incendie, le vol de certains objets, la blessure de l'infirmier-chef. En revanche, quel statut accorder aux morts successives de Rocket, d'Amber et de Blondie? Eu égard à son attitude professionnelle dans la réalité, où elle fait appel à la police pour démasquer les agissements de l'infirmier-chef, il est peu vraisemblable que Vera Gorski ait pu taire les meurtres de son comparse Blue, ce qui tendrait à confirmer que ceux-ci sont le fruit de la fiction intérieure élaborée par Babydoll. Aussi la structure de dissimulation des faits de la mise en scène met-elle le spectateur en demeure d'enquêter pour distinguer les éléments qui ont une fonction de refiguration de ceux qui sont purement fantasmés. 
À partir de ce point, le "voilement» dans Sucker Punch conditionne un possible "dévoilement» qui ne se résout que par un acte de reconstitution du sens. Le récepteur est convié à élaborer la signification de ce qui lui est montré, à rebours des emboîtements diégétiques. Cette archéologie des images permet d'établir des rapports d'isomorphisme entre les différents niveaux. L'association sémantique des violences de l'asile avec la danse, elle-même figurée comme combat, opère le rapprochement entre la réalité de l'asile et le monde de la fantasy: les séquences guerrières évoquent la lutte des patientes pour faire face à ce qu'elles subissent; dans la perspective où chaque niveau élève la puissance (au sens mathématique du terme) expressive du délire, la danse représente, par hyperbole, les violences guerrières dont elles sont les héroïnes et, par euphémisme, les abus dont elles sont les victimes.

Plusieurs procédés invitent à "enquêter» sur le sens du récit. Le réalisateur met au point un plan qui renverse, de façon vertigineuse, la perspective de la situation filmée: un travelling qui fait pénétrer dans le reflet des événements - lequel se substitue définitivement à la réalité 7 . Ce procédé inverse la subordination du reflet au réel et disqualifie l'authenticité des images. On peut repérer également le motif du paradis, relayé par la présence récurrente d'une bouteille d'alcool dont l'étiquette porte le nom «Paradise». Ce terme désigne à la fois l'état catatonique du sujet ayant subi une lobotomie («Elle sera au paradis. Vous voyez ce que je veux dire?») et l'extérieur, le monde hors des murs de l'asile (ce qu'évoque le panneau «Paradise Diners» dans la dernière image). Ces deux acceptions entretiennent un lien de contiguïté en ce que le "paradis» constitue l'horizon tant négatif que bénéfique des patientes, une sorte de non-lieu qui signifie l'ambiguïté fantasmatique; par corollaire, la bouteille manifeste l'intrusion de l'irréel dans ce qui est montré (on voit un gros plan sur cette bouteille lorsque Blue «tue» Amber). Un dernier mécanisme, qui repose sur la reprise, par les acteurs eux-mêmes, de chansons connues, invite à examiner les interférences entre la bande musicale et la narration. La plupart des paroles chantées thématisent l'indistinction du rêve et de la réalité, soit de façon explicite, comme celles de Where Is My Mind?, soit de façon 
implicite en contredisant les faits, comme la répétition de la phrase "It is not dying" de Tomorrow Never Knows qui scande la séquence de la mort de Rocket.

Sucker Punch entretient donc la confusion en ce qui concerne la distinction entre la réalité et le fantasme. Or, d'emblée, les premières images du film instaurent de manière originale ce que Philippe Lejeune (1975) appelle un "pacte de lecture», fondé sur une mise en abyme de la théâtralité. Les logos des sociétés de production s'inscrivent sur le rideau rouge d'un théâtre, pendant que la caméra opère un travelling des rangs du public vers la scène. Ce cadre révèle la "mise en scène" avec laquelle le point de vue du narrateur fusionne pour devenir le degré zéro de la narration. Lors de ce processus, une voix off énonce "ne vous fiez pas aux apparences", invitant ainsi les spectateurs à adopter une posture critique. De la sorte, le réalisateur thématise la fictionnalité des images, en révélant la coulisse de la narration, loin de la "feintise ludique partagée " de Jean-Marie Schaeffer (1999).

Que tend à "dévoiler» la narration à l'aide de ces indices? L'analyse du titre, dont le potentiel sémique repose sur l'association d'un jeu graphique et d'un signifié verbal, s'avère à cet égard éclairante. Le titre combine l'idée d'attaque (punch) avec celle de transgression (sucker, qui ici évoque tant l'imprévu que la bassesse), et met en avant l'idée de surprise. La majuscule du mot «Sucker» est traversée par le "pic à glace» qui sert à pratiquer une lobotomie. Le titre invite ainsi à interroger l'effet de surprise par rapport à cette opération. De fait, Zack Snyder signifie au spectateur qu'il dissimule quelque chose en prenant soin de cacher après l'intervention le visage de Babydoll — lors même que le spectateur sait qu'il s'agit d'elle. Le réalisateur entretient un suspense surprenant qui tend à investir le visage de l'héroïne d'une signification d'autant plus importante qu'elle ne s'avoue pas. On comprend que le rapport à l'image que propose Zack Snyder conduit à une "suspension consentie de la crédulité ${ }^{8}$ " à l'égard du donné visuel. Sous couvert d'une structure qui opère un va-et-vient mécanique entre imaginaire et réalité, Sucker Punch invite à considérer son apparentement au genre du mindfuck ${ }^{9}$ — c'est-à-dire des films qui surprennent le spectateur 
par des chausse-trapes interprétatives - et donc à effectuer une relecture des incohérences au profit d'une signification dérobée.

\section{La subjectivité comme lieu du secret}

Lacte de "déchiffrement» auquel est convié le spectateur revient à élaborer un récit second à partir des éléments narratifs qui lui sont donnés. Il s'agit d'adopter la perspective de Pierre Bayard (1998), en considérant que l'œuvre aveugle d'une certaine façon le sujet de la réception et en s'employant à déconstruire le discours de l'œuvre pour formuler un autre "délire» à visée explicative:

Entreprendre d'édifier un délire présente en tout cas un avantage: permettre de réfléchir autrement sur la nature ou les fondements d'une lecture vraie (Bayard 1998, p. 15).

Snyder offre la possibilité d'une multiplicité de lectures fondée sur la mise en abyme de la théâtralité. La salle de spectacle qui constitue le seuil du récit s'avère la même que celle où se produisent les danseuses, laquelle n'est elle-même que la transfiguration de la salle commune de l'institut Lennox — surnommée "Le Théâtre" - où ont lieu les mises en scène à visée cathartique du vécu des patientes. Cette thématique fonde l'ambivalence de la notion de «libération" en tant qu'elle implique l'évasion au double sens de sortie hors d'un lieu clos et d'exclusion du "principe de réalité ${ }^{10}$ ". La question se pose de savoir quelle libération se trouve exactement mise en jeu: est-ce symboliquement ou réellement que Sweet Pea recouvre sa liberté à la fin de l'histoire ${ }^{11}$ ?

Cette ambivalence repose, pour une part, sur celle du concept de "paradis " qui relie en miroir le destin de Sweet Pea à celui de Babydoll. Celle-ci jouit d'un "paradis» intérieur à la suite de la lobotomie, c'est-à-dire qu'elle bénéficie d'une absence à soi qui la rend insensible aux sévices, tandis que celle-là, seule à être parvenue à s'évader physiquement du cabaret-bordel, s'éloigne dans un "paradis" tangible. Leur libération respective les lie intimement l'une à l'autre, car Babydoll doit se sacrifier pour rendre possible l'évasion de Sweet Pea: elle doit consentir à la lobotomie pour libérer son amie. Cette intrication des deux 
destins explique en partie la réaction étonnée du médecin après la lobotomie: "Je n'ai jamais vu quelqu'un... La façon dont elle m'a regardé... Comme si elle voulait que je le fasse. " Aussi savoir quelle libération se trouve mise en jeu implique-t-il de déterminer qui est libéré.

Cette problématique identitaire est l'objet, de façon sousjacente, d'un questionnement phénoménologique. Dès l'ouverture, le film rappelle que le cinéma repose sur le partage du point de vue d'un narrateur. L'identification du cadre de la caméra au regard du spectateur fait de chaque plan l'expression d'une subjectivité en tant que perspective choisie par le réalisateur. Cela rejoint la pensée de Michel Henry (2000), pour qui tout vécu se fonde singulièrement sur l'épreuve pathétique de soi, en tant quauto-donation de la $\mathrm{Vie}^{12}$. Ainsi, la problématique distinction entre le réel et l'imaginaire dans Sucker Punch souligne les limites phénoménologiques de la réalité : il n'y a pas de fondement d'une objectivité réelle pour Michel Henry, étant donné que la "chair» constitue la condition de possibilité de toute expérience. Toute narration est régie par le filtre d'une subjectivité qui ne s'avoue pas, de sorte que le film se conçoit comme la mise en jeu d'un soi fictif. Dès lors, il s'agit de déterminer quelle subjectivité se met à l'œuvre.

Le réalisateur subvertit d'emblée le code qui prescrit d'élever le personnage principal au rang de héros en faisant de son "héroïne» un leurre. Babydoll correspond à la description des anges énoncée par la voix off du début — anges qui peuvent notamment prendre la forme d'une petite fille et dont le "rôle n'est pas de livrer nos batailles, mais de souffler dans nos cœurs " pour nous pousser à lutter, en nous rappelant que «nous contrôlons les mondes" que nous créons. Par la suite, son rôle d'ange est confirmé par le fait qu'elle pousse ses compagnes à lutter pour s'évader. On notera par ailleurs l'inconsistance de son personnage au début du film, où elle ne prononce pas la moindre parole - à l'opposé de Sweet Pea qui assume le rôle de narratrice homodiégétique. Pour finir, elle choisit de se sacrifier dès qu'elle comprend que la solution de l'énigme qui mène à la libération ( La cinquième est une énigme. C’est la raison. Le but. Ce sera un grand sacrifice et une victoire parfaite. Toi seule peux 
la résoudre. Si tu y arrives, tu seras libérée») se trouve être ellemême: "C'est moi. C'est moi, évidemment. C'est la seule fin possible." Babydoll est conçue comme un personnage secondaire, un adjuvant; ce que confirme cette confidence finale faite à Sweet Pea : "Ça n'a jamais été mon histoire. C'est la tienne.»

En contrepartie, il apparaît que tout se noue autour de la figure de Sweet Pea, qui se révèle être la véritable héroïne et le seul sujet de la quête d'évasion. C'est elle en effet qui lance de façon théâtrale, dès le début du récit dans le cabaret-bordel, qu'elle est "la vedette du spectacle» — à entendre plus largement «du film». Sur le mur de la salle où se préparent les danseuses se trouve une affiche dont le slogan affirme "My dream is yours". Alors que la narration, de façon trompeuse, place Babydoll au centre des événements et en fait la source des mondes imaginaires, cette phrase décentre cette perspective au profit d'un "tu» — Sweet Pea. Un dernier détail permet d'accréditer cette hypothèse : le sigle « $\mathrm{S}$. P.» que portent les policiers qui menacent d'arrêter Sweet Pea dans la dernière scène fait écho aux initiales du nom de la jeune fille — initiales qui sont également celles du titre du film.

D'où il appert que l'existence de Babydoll est illusoire, purement fantasmée, comme l'indique l'échange des regards lors de la première rencontre entre Babydoll et Sweet Pea, ceux-ci se croisant au moment où Vera Gorski dit à Sweet Pea: "Tu contrôles ce monde [...] Ce que tu es en train d'imaginer, ce monde que tu contrôles, ce lieu peut être aussi vrai qu'une douleur." La scène se passe sur le fond musical de la chanson intitulée de façon significative Where Is My Mind? Autant d'indications qui subordonnent l'existence de Babydoll au regard démiurgique de Sweet Pea qui enrichit le réel par ses projections. En outre, le dialogue du début du film entre le beau-père et l'infirmier introduit le thème du double: Babydoll apparaît en gros plan, le visage à moitié coupé par la caméra tandis que soit l'un soit l'autre apparaît dans le champ au second plan, selon un effet de symétrie. Babydoll se révèle être une sorte d'hétéronyme de Sweet Pea, qui s'autonomise dans les mondes imaginaires.

L' «identité » des deux personnages se fonde sur un jeu de symétries qui constituent autant d'indices de leur substituabilité. 
On peut souligner la proximité entre l'incipit et la scène où Babydoll entre dans "Le Théâtre ": les deux héroïnes se trouvent dans la même posture, ce qui suggère que la scène introductive est la projection d'un souvenir remanié de Sweet Pea. Leur interchangeabilité apparaît encore davantage dans le passage du réel au premier niveau imaginaire. Ce monde s'insère dans le récit comme une analepse (le film revient sur le passage de Babydoll dans "Le Théâtre») et opère une dilatation temporelle (le temps de ce récit enchâssé condense cinq jours en la fraction de seconde que dure l'impact de l'aiguille). La caméra opère un travelling sur le visage de Babydoll, menacée par le pic à glace, jusqu'à ce qu'une voix crie "Stop!» La caméra recule: le passage dans l'imaginaire vient de se produire et c'est Sweet Pea qui se trouve dans le fauteuil, déguisée en Babydoll. Or, à bien regarder les gros plans des yeux dans cette séquence, on peut voir que ceux de Sweet Pea sont déjà substitués, dans l'asile, à ceux de Babydoll. Ce passage souligne la possibilité de fondre les deux personnages en un seul.

Cette lecture permet d'interpréter à nouveaux frais les incohérences scénaristiques. La percussion des différentes sphères de réalité dans la dernière scène indique que le vécu de la jeune fille permet de relier ces différents personnages, en tant que centresource de la narration. En outre, si l'on ne peut douter de la réalité des trois protagonistes qui complètent l'équipe, celles-ci incarnent autant d'hétéronymes de Sweet Pea: ce sont leurs relations qui sont fantasmées, ce qui explique pour une part leur manque de personnalité, qui les assimile à des adjuvants sans épaisseur. Cependant, le rôle de Babydoll est axiologiquement et structurellement plus fondamental. En effet, Sucker Punch se comprend comme le récit de la libération de Sweet Pea qui passe par le truchement d'une mise à distance partielle de son identité. Tout ce que vit Babydoll renvoie à son propre vécu. Dès lors, l'imaginaire dans lequel s'évade Sweet Pea / Babydoll se conçoit comme un moyen d'échapper à la culpabilité d'avoir tué accidentellement sa sœur, de sorte que le délire vise à négocier une souffrance existentielle par le biais d'une fiction à visée réparatrice. Sweet Pea se décharge du poids de cet acte par la mise en œuvre d'un récit qui l'accuse elle-même comme un 
autre, de façon schizoïde. L'initiation à laquelle aboutit Sucker Punch correspond donc au sacrifice du double de Sweet Pea, en tant que geste symbolique qui la décharge de la culpabilité. Le "paradis" se révèle ainsi le lieu d'une ouverture sur un véritable horizon à l'avant d'elle — symbolisé par la route qu'emprunte le bus dans un paysage baigné par la lumière du soleil —, c'està-dire loin du passé, qui lui permet de se construire une nouvelle vie.

Par une schize du moi, Sweet Pea peut imaginairement laisser Babydoll prendre sa place extérieurement sur la chaise d'opération pendant qu'elle s'évade intérieurement en se recréant un monde qu'elle peut habiter sereinement ${ }^{13}$. On comprend pourquoi le réalisateur prend soin de dissimuler le regard de Babydoll après la lobotomie: ce procédé rend attentif aux répliques à double entente de l'infirmier-chef qui, surpris dans ses agissements, tente de se justifier: «Regardez-la! Elle est partie.» Cette assertion évoque tant une absence métaphorique liée à la lobotomie qu'une véritable absence du personnage. La caméra à ce moment opère un panoramique pour passer de Babydoll sur sa chaise, dans les toilettes sombres des hommes, à Sweet Pea qui, elle, sort des toilettes des femmes - ce qui souligne une dernière symétrie où se joue la réparation symbolique des violences dont elle peut faire l'objet. C'est par ailleurs dans ce monde intérieur que se retrouvent les seules figures masculines positives: le jeune soldat croisé dans les tranchées et le Sage, reconverti en chauffeur de bus.

In fine, la nécessité d'élaborer un récit interprétatif pour déployer les virtualités de Sucker Punch montre que l'œuvre de Snyder, au-delà de sa charge visuelle transgressive, déploie une esthétique du secret. Le film ne cesse de dérober le visible au spectateur; de sorte que le secret se conçoit comme l'inaccessibilité d'un sens intentionnel qui ouvre le champ à de multiples possibles. Cela rejoint l'approche de la lecture de Michel Lisse (2001, p. 135), pour qui «le secret exemplaire de la littérature tient dans cette impossibilité de lire autre chose que le secret». Ainsi Sucker Punch met-il le spectateur au défi de le décoder pour le «voir» véritablement. 
Au terme de cette analyse, on en vient à penser que Sucker Punch doit son faible succès à son caractère atypique au sein du paysage cinématographique actuel. L'œuvre s'avère transgressive, à la fois sur le plan esthétique - en proposant un décloisonnement des genres sur le mode de l'intertextualité - et sur le plan formel - par la remise en question du code qui interdit de s'avouer l'expression d'une "subjectivité radicale" (Henry 2000). Le film déçoit l'attente des spectateurs par une esthétique du secret qui les place malgré eux en un lieu où il témoigne de ce que son horizon de sens ne se dévoile jamais. En d'autres termes, l'œuvre ne permet à aucun moment de déterminer la réalité de ce qui est montré: pur produit d'un narrateur, chaque élément peut s'avérer illusoire, constitutif d'une fiction qui est mensonge ludique.

Cette approche implique un renversement des enjeux du cinéma: non pas tout révéler, dans l'illusion d'une transparence des images, mais rappeler qu'il s'agit — à l'instar de la poésie moderne - de "faire l'épreuve que [la chose] se dérobe", ce qui «suppose d'affronter et d'accepter la perte de l'objet comme la condition même de l'œuvre où il reparaîtra transfiguré " (Collot 1989, p. 148). Ce qui fait écho à la dédicace discrète à la mère décédée du cinéaste ${ }^{14}$ sur laquelle le film se clôt, en ce sens que le secret qui sous-tend la narration manifeste la pudeur du réalisateur à l'égard d'un deuil à accomplir — l'histoire s'ouvrant de façon significative sur la mort de la mère de Babydoll. Ainsi le trajet de Sweet Pea est-il assimilable à celui du sujet filmant qui, par l'entremise de l'art, tente de surmonter la perte d'un proche.

Sucker Punch, en tant qu'accomplissement d'une thérapie fondée sur le pouvoir de l'imaginaire - lieu de réparation des blessures narcissiques pour rendre le monde habitable déploie de façon sous-jacente une réflexion sur le septième art. Car l'évasion thématisée dans Sucker Punch concerne, en dernière instance, le spectateur lui-même:

Et enfin, cette question: qui sera au centre de l'histoire? Qui baisse le rideau? Qui choisit les pas que l'on danse? Qui nous rend fous? Nous fouette et nous auréole de victoire quand on 
survit à l'impossible? Qui donc... fait toutes ces choses? [...] Qui honore ceux qu'on aime pour la vie qu'on mène? [...] C'est vous. Vous avez toutes les armes qu'il vous faut. Alors, battez-vous.

L'œuvre de Snyder offre un supplément d'existence, dès lors que l'indistinction problématique entre le réel et l'imaginaire s'abolit sur le plan de l'affectivité. Libéré des contraintes d'un rapport «réaliste» au réel, le cinéma peut alors déployer toutes ses possibilités d'enrichissement de l'imaginaire et, de la sorte, induire la libération momentanée des contingences quotidiennes.

\section{Université catholique de Louvain}

\section{NOTES}

1. Voir la conférence intitulée "Dans l'ombre du Cid: (in)fortune de L'Illusion comique", donnée par Jonathan Decubber dans le cadre d'un séminaire doctoral sous la direction d'Agnès Guiderdoni à l'Université catholique de Louvain le 8 décembre 2011 (http://gemca.fltr.ucl.ac.be/php/evenements/20111208.php); voir aussi Decubber 2014.

2. On notera à ce propos que la reprise «intertextuelle» explicite que pratique ouvertement Zack Snyder dans ce film figure en bonne place parmi les critiques qui lui ont été adressées.

3. Cette lecture privilégie l'analyse interne du film; dans les limites de cette investigation, on ne développera pas la question, très présente cependant, de la fétichisation des personnages féminins, qui touche à des éléments d'ordre sociologique et qui invite à considérer le film comme le lieu où s'opère la radiographie des imaginaires féminins à un moment de l'histoire cinématographique.

4. Zack Snyder s'inspire librement, dans une scène, de l'univers du Seigneur des anneaux (1954-1956) de John Ronald Leuen Tolkien, adapté au cinéma par Peter Jackson (The Lord of the Rings, 2001-2003).

5. Ces éléments témoignent de l'influence importante du film The Matrix (Matrix / La matrice, 1999) de Andy et Lana Wachowski (voir Dubois 2011).

6. Son nom, composé des mots baby et doll, laisse entendre à la fois un hypocoristique réducteur pour son statut d'adolescente et sa chosification en jouet.

7. Ces plans font référence à la suite d'Alice au pays des merveilles, intitulée De l'autre côté du miroir et publiée par Lewis Caroll en 1872.

8. L'expression est dérivée de la célèbre formule de Samuel Taylor Coleridge — «suspension consentie de l'incrédulité» (willing suspension of disbelief) — dans sa Biographia Literaria (1817).

9. La spécificité du mindfuck repose sur un twist final, un retournement qui vient surprendre le spectateur, amené à élaborer jusque-là une interprétation erronée et plus triviale que ce que le film en réalité déploie.

10. Le "principe de réalité», théorisé par Freud, se comprend ici comme force qui tient compte des exigences du réel et extrait le sujet du monde de l'hallucination et du rêve, où triomphe à l'opposé le "principe de plaisir ». 
11. Cette perspective est à nuancer au regard de la fétichisation des héroïnes, dès lors qu'elle tend à les réduire à des stéréotypes. Ce traitement des personnages est lié, entre autres, au plaisir de réaliser une œuvre qui brasse une multitude de codes ancrés dans la culture populaire des films d'action et des jeux vidéo, dans un rapport ambivalent à la fois de distanciation - marqué par leur caractérisation outrancière — et d'adhésion.

12. «L'Archi-intelligibilité appartient au mouvement interne de la Vie absolue qui s'engendre elle-même, n'étant rien d'autre que la façon selon laquelle ce procès d'auto-engendrement s'accomplit. [...] Or aucune épreuve de soi n'est possible si n'advient en elle, en même temps qu'elle et comme sa condition, une Ipséité qui lui est ainsi consubstantielle. Ipséité désigne le fait d'être soi-même, le fait d'être un Soi» (Henry 2000, p. 29).

13. La seule chose qui affaiblit cette interprétation est la mention qu'une patiente s'est réellement évadée de l'asile. Toutefois, la mise en scène elliptique du film ne certifie en rien qu'il s'agit bien de Sweet Pea et autorise une lecture selon laquelle une autre patiente, que l'on ne connaît pas, aurait profité de l'incendie provoqué par Sweet Pea / Babydoll pour s'enfuir.

14. On peut lire à la fin du générique: «In loving memory of Marsha Snyder 1935$2010 »$.

\section{RÉFÉRENCES BIBLIOGRAPHIQUES}

Bayard 1998: Pierre Bayard, Qui a tué Roger Ackroyd?, Paris, Minuit, 1998.

Collot 1989: Michel Collot, La poésie moderne et la structure d'horizon [1989], Paris, PUF, 2005.

Decubber 2014: Jonathan Decubber, "L'Illusion comique en France par Giorgio Strehler (1984) et Galin Stoev (2008). Proposition pour un outil d'appréhension des phénomènes de réception", Les Lettres romanes, vol. 68, n 3-4, 2014, p. 525-537.

Dubois 2011 : Matthieu Dubois, «De l'expérience pathétique de la fiction visuelle à la lecture des signes littéraires. Analyse de The Matrix et d'Avatar à partir de Michel Henry", Echinox, n' 20, 2011, p. 177-188.

Durand 1960 : Gilbert Durand, Les structures anthropologiques de l'imaginaire. Introduction à l'archétypologie générale [1960], Paris, Dunod, 1997.

Eco 1979: Umberto Eco, Lector in fabula ou La coopération interprétative dans les textes narratifs [1979], Paris, Grasset, 1985.

Ferrer 1998: Daniel Ferrer, "Le matériel et le virtuel: du paradigme indiciaire à la logique des mondes possibles", dans Michel Contat et Daniel Ferrer (dir.), Pourquoi la critique génétique? Méthodes, théories, Paris, CNRS Éditions, 1998, p. 11-30.

Henry 2000 : Michel Henry, Incarnation. Une philosophie de la chair, Paris, Seuil, 2000.

Lejeune 1975: Philippe Lejeune, Le pacte autobiographique, Paris, Seuil, 1975.

Lisse 2001 : Michel Lisse, L'expérience de la lecture 2. Le glissement, Paris, Galilée, 2001.

Schaeffer 1999 : Jean-Marie Schaeffer, Pourquoi la fiction?, Paris, Seuil, 1999.

Snyder 2011 : Romain Le Verne, «Sucker Punch: Interview Zack Snyder», MyTF1.fr, 30 mars 2011 (http://lci.tf1.fr/cinema/news/sucker-punch-interview-zack-snyder6333130.html).

Watthee-Delmotte 2010 : Myriam Watthee-Delmotte, Littérature et ritualité. Enjeux du rite dans la littérature française contemporaine, Bruxelles, P.I.E. Peter Lang, 2010.

Incohérence et transgression dans Sucker Punch: une esthétique du secret. Lecture phénoménologique d'un (dé)voilement du sens 


\section{ABSTRACT}

\section{Incoherence and Transgression in Sucker Punch: An Aesthetic of the Secret. A Phenomenological Reading of an (un)Veiling of Meaning Matthieu Dubois}

This article takes up the task of reading Zack Snyder's Sucker Punch (2011) against the grain of those critics who saw in it only a film consumed by contradictions and a shallow script. Beginning with an analysis of diegetic transport, the author highlights the reasons he has developed an against-the-grain interpretation of the narrative based on Pierre Bayard's (1998) analysis of Agatha Christie's The Murder of Roger Ackroyd. With the assistance of Michel Henry's phenomenology, the author updates the necessarily subjective and deceptive nature of filmic narration, inviting us to see a twist in the structure of the film's story: that the existence of the heroine (Babydoll) is illusory in favour of a secondary character (Sweet Pea), in a personality doubling process enacted to distance a traumatic experience. This makes Sucker Punch a work that explores deeply the potentialities of the imagination and offers a kind of negative reflection of the issues at play in cinema in the present era, as digital effects grow without precedent. 